\title{
The Role of Peripheral N-Methyl-D-Aspartate (NMDA) Receptors in Freund's Complete Adjuvant Induced Mechanical Hyperalgesia in Rats
}

\author{
Tae Ha Lim, M.D., Seung Sig Kang, M.D., Jun Gol Song, M.D. \\ and Yoon Choi, M.D. \\ Department of Anesthesiology, University of Ulsan, College of Medicine, Seoul, Korea
}

- Abstract $=$

Background: While the effects of excitatory amino acids have been characterized in the central nervous system, relatively little is known about their possible modulation of elements responsible for hyperalgesia within peripheral tissue. The purpose of this study was to investigate the role of excitatory amino acid receptors in mechanical hyperalgesia induced by a subcutaneous injection of Freund's complete adjuvant (FCA) into the rat hind paw.

Methods: Inflammations were induced by injecting FCA on the dorsal surface of the right hind paw of rats. Effects of excitatory aminoacid agonists or antagonists on mechanical hyperalgesia were investigated by a subcutaneous injection of a drug to the inflamed paw. Mechanical hyperalgesia was expressed as percent change in paw withdrawal threshold compared to baseline value that was measured before drug injection after inflammation was induced with FCA.

Results: In normal rats, an intraplantar (i.pl.) injection of L-glutamate, but not of D-glutamate (3 pmol/0.1 ml each) produced a mechanical hyperalgesia in the hind paw with a lowered paw pawwithdrawal threshold to pressure. In rats that developed the mechanical hyperalgesia associated with inflammation in the hind paw following an i.pl. injection of FCA $(0.15 \mathrm{ml})$, the injection of a N-methyl-D-aspartate (NMDA) receptor antagonist, MK-801 (1 pmol/0.1 ml) into the inflamed paw increased the paw pressure threshold $(24.24 .6 \%$ increase from baseline, $P<0.05)$. On the other hand, the injection of a non-NMDA receptor antagonist, 6-cyano-7-nitroqiunoxaline-2,3-dione (CNQX, 10 pmol/0.1 ml) into the inflamed paw had no effect on the FCA-induced lowering of the paw pressure threshold.

Conclusions: The results suggest that NMDA, but not non-NMDA receptors play a substantial role in mediating the development of mechanical hyperalgesia induced in the inflamed paw following an i.pl. FCA injection. (Korean J Anesthesiol 2000; 39: S 35 S 40)

Key Words: Antagonist, uiscellaneous: MK-801; CNQX. Pain: experimental; inflammatory. Receptor: NMDA; Non-NMDA.

Received : November 22, 2000

Corresponding to : Y Choi, Department of Anesthesiology, College of Medicine, University of Ulsan, 388-1 Poongnap-dong, Songpa-gu, Seoul, Korea.

Tel: 82-2-2224-3868, Fax: 82-2-470-1363, E-mail: ychoi@www.amc.seoul.kr 


\section{INTRODUCTION}

Existence of glutamate receptors in peripheral nerves innervating normal ${ }^{1,2)}$ and inflamed skin $^{3,4)}$ has been well addressed. An involvement of glutamate receptors in peripheral nociception has been demonstrated on animal models of inflammatory pain induced by subcutaneous injection of formalin ${ }^{5)}$ or carrageenan. ${ }^{6,7)}$ Freund's complete adjuvant (FCA) has been used as another inflammation-producing chemical, the Freund's complete adjuvant (FCA) has been used to produce responses that mimic human clinical pain condition in which the pain lasts for longer periods of time than when induced by these other agents. The injection of FCA into the rat hind paw has been shown to produce mechanical hyperalgesia that accompanies long-lasting inflammation in the injected hind paw. ${ }^{8)}$ A recent study has demonstrated that the number of peripheral primary afferent axons expressing glutamate receptors is significantly increased during FCA-induced inflammation of the rat hind paw. ${ }^{3)}$ However, it has not yet been proven whether the peripheral glutamatergic system is involved in mediating mechanical hyperalgesia accompanying FCA-induced inflammation. The present study was performed to examine whether the injection of glutamate into the rat hind paw evokes mechanical hyperalgesia, and whether glutamate receptor subtypes contribute to the mechanical hyperalgesia observed in the inflamed hind paw induced by FCA.

\section{MATERIALS AND METHODS}

The protocol of the present study was approved by the Institutional Animal Investigation Committee. Male Sprague-Dawley rats $(200-250 \mathrm{~g})$ were housed in groups of four with food and water available ad libitum under a light-dark cycle. All animals were habituated to the behavioural testing for about aapproximately one week before the beginning of data collection. Mechanical hyperalgesia was evaluated by measuring the paw-withdrawal threshold to pressure using an automated gauge (Ugo Basile, Italy). Incremental pressure was applied via using a round-shaped tip of a piston (4 mm of tip radius) onto the dorsal surface of the hind paw and the pressure required to elicit paw withdrawal, the paw pressure threshold, was determined. Four consecutive trials, separated by intervals of $15 \mathrm{sec}$, were conducted at predetermined sites on the dorsum of the hind paw: over the center of the dorsum, at the distal end of the $5^{\text {th }}$ fifth metatarsal, at the interspace of the distal ends of the $3^{\text {nd }}$ third and the $4^{\text {th }}$ fourth metatarsals, and at the distal end of the $2^{\text {nd }}$ second metatarsal bones. The data obtained from these four measurements were averaged and used as a value for the paw paw-pressure threshold.

After baseline paw-pressure thresholds were obtained, an intraplantar injection was made into one hind paw with L-glutamate (3 nmol/0.1 ml), D-glutamate (3 nmol/0.1 $\mathrm{ml}$ ), or vehicle control phosphate buffered saline (PBS, $0.1 \mathrm{ml}$ ). Paw-pressure thresholds were measured at 15,30 , 60,90 and 120 mins after injection of each drug. A comparison was made among three cases.

Inflammation was induced by an intraplantar injection of $0.15 \mathrm{ml}$ FCA (Sigma Aldrich) into the one hind paw under enflurane anesthesia (4\% enflurane- $\mathrm{O}_{2}$ mixture). Two days after FCA injection, when characteristic signs of inflammation were observed, either the non-competitive NMDA receptor antagonist MK-801 (0.1 or 1 pmol/0.1 $\mathrm{ml}$ ), the competitive AMPA/kainate receptor antagonist 6-cyano-7-nitroqiunoxaline-2,3-dione (CNQX) (1 or 10 $\mathrm{pmol} / 0.1 \mathrm{ml})$, or PBS $(0.1 \mathrm{ml})$ was injected into the plantar surface of the inflamed hind paw. To see if the effect of the intraplantar injection of MK-801 or CNQX could be via centrally mediated mechanisms, this effect was compared to the effect exerted by subcutaneous injection of the same drug into the rat's back skin. All drugs (purchased from Sigma Aldrich) were dissolved in PBS (pH 7.4) and injected under a brief enflurane anesthesia. Paw-pressure thresholds were measured before and $15,30,60,90$ and $120 \mathrm{~min}$ after the drug's injection.

All data are presented as mean \pm standard errors of the mean. Analysis of variance was used to determine statistical differences between the groups according to the experimental design. Duncan's multiple range test was used for post-hoc comparisons between groups. 


\section{RESULTS}

The time course of changes in the paw paw-pressure threshold following an intraplantar injection of glutamate in the normal paw is shown in Fig. 1. Measurement was made on the injected paw. Before injection, average baseline paw-pressure threshold across all groups was $283.1 \pm$ $17.8 \mathrm{~g}$ ( $\mathrm{n}=10$ for each group). The injection of $3 \mathrm{pmol}$ of glutamate reduced the paw-pressure threshold gradually as time went byover time, until 120 minutes post-injection. However, when these changes were compared to threshold changes following PBS injection, the difference was statistically significant at $\mathbf{1 5}$ and $\mathbf{3 0}$ minutes post-injection $(\mathrm{P}<0.05): 10.5 \pm 3.2 \%$ reduction vs. $4.2 \pm 6.0 \%$ increase from baseline levels at 15 minutes post-injection and $12.7 \pm 2.8 \%$ reduction vs. $3.4 \pm 3.7 \%$ increase from baseline at 30 minutes post-injection. To examine whether glutamate's effect was receptor mediated, threshold change

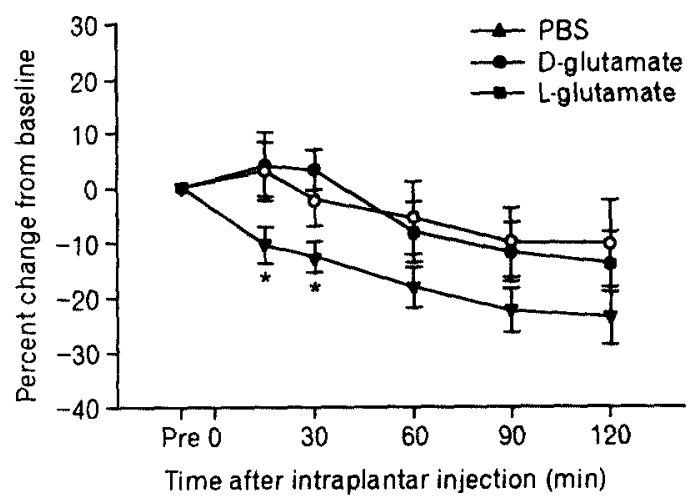

Fig. 1. Time course of changes in mechanical nociception following an intraplantar injection of glutamate into normal tissue. Baseline values for the paw-withdrawal threshold to pressure were measured before local intraplantar injection (at 0 ) of L-Glutamate ( $3 \mathrm{pmol}$ ), D-Glutamate (3 pmol) or PBS $(0.1 \mathrm{ml})$ into the rat hind paw. Measurement was repeated at 15, 30,60,90 and 120 minutes post-injection. Data represent the percent change of the paw-pressure threshold from baseline. Average baseline paw-pressure threshold across all groups was $283.1 \pm 17.8 \mathrm{~g}$ ( $\mathrm{n}=10$ for each group). Error bars are S.E.M. *: $P<0.05$, significantly different from the PBStreated control. was also tested with the metabolically inactive stereoisomer D-glutamate. D-glutamate injection tended to change the paw-pressure threshold; however, this effect was not statistically different from that observed in the PBS-treated control.

The effect of an intraplantar injection of antagonist for NMDA or non-NMDA receptor on FCA-induced mechanical hyperalgesia was investigated. We have observed during FCA-induced inflammation that the paw-pressure threshold decreased with a peak reduction $(44.5 \pm 4.7 \%$ change from baseline levels of $279.5 \pm 16.7 \mathrm{~g}, \mathrm{n}=10$ ) on day 2 and then slowly subsided with the reduced threshold maintaining until 8 the eighth weeks of the observation period. Thus, an intraplantar injection of MK801 or CNQX into the inflamed paw was carried out 2 days after FCA injection and paw-pressure thresholds were measured thereafter. These results are presented in Fig. 2. As seen in Fig. 2A, where average baseline paw-pressure threshold across all groups was $155.2 \pm 13.4 \mathrm{~g}$ ( $\mathrm{n}=15$ for each group), the administration of 1 pmol of MK-801 tended to increase paw-withdrawal thresholds as compared to the PBS-treated control group, but a statistically significant difference was observed at 15 minutes post-injection (24.2 $\pm 4.6 \%$ vs. $5.4 \pm 3.3 \%$ increases from baseline, $P<0.05$ ). At this time point, the antinociceptive effect of MK-801 was dose-dependent (14.9 $\pm 3.0 \%$ and 24.2 $\pm 4.6 \%$ increases for 0.1 pmol and 1 pmol, respectively). This effect appears not to be centrally mediated since the subcutaneous injection of 1 pmol MK-801 into the animal's back skin produced changes in the paw-pressure threshold that were not significantly different from those observed with the intraplantar injection of PBS.

CNQX was also evaluated in the same paradigm for peripheral antinociceptive effect. As seen in Fig. 2B, where average baseline paw-pressure threshold across all groups was $150.0 \pm 9.7 \mathrm{~g}$ ( $\mathrm{n}=10$ for each group), the intraplantar injection of CNQX, even at a dose of $10 \mathrm{pmol}$, failed to show a statistically significant difference in the paw-pressure threshold at all time points as compared to the PBS-treated hind paw. No significant difference in the paw-pressure threshold was observed between groups injected with 10 pmol CNQX into the hind paw and those 

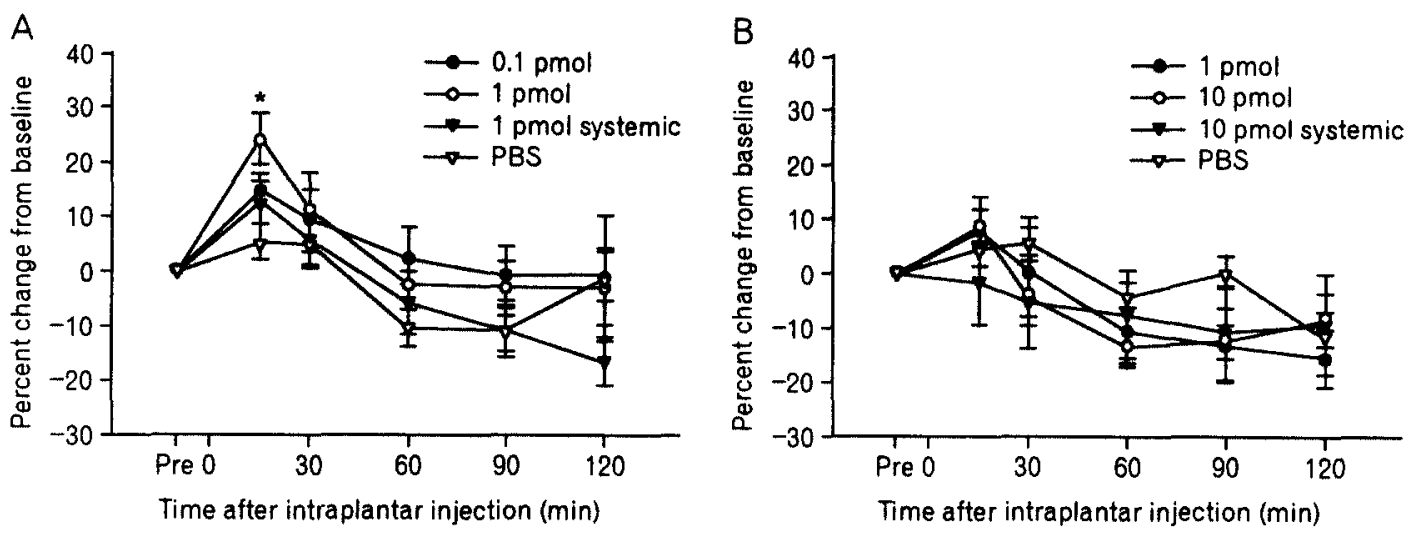

Fig. 2. Effects of MK-801 or CNQX on FCA-induced mechanical hyperalgesia. Inflammation was induced by an intraplantar injection of FCA $(0.15 \mathrm{ml})$ into the rat hind paw. Two days after FCA injection, baseline paw-pressure threshold was measured, followed by local administration of MK-801, CNQX or PBS vehicle into the inflamed hind paw (at 0). Measurement was repeated at 15, 30, 60, 90 and 120 minutes after drug administration. Panel A shows the time course of changes in the pressure threshold following local injection of MK-801 (0.1 or 1 pmol). Possible systemic effects of 1 pmol of the intraplantar MK-801 were evaluated with an equivalent dose of MK-801 applied subcutaneously into the rat's back skin for another group. Data represent the percent change of the paw-pressure threshold from baseline. Average baseline paw-pressure threshold across all groups were $155.2 \pm 13.4 \mathrm{~g}$ ( $\mathrm{n}=15$ for each group). Panel B shows the results from experiments done in the same paradigm as above using CNQX $(0.1$ or $1 \mathrm{pmol})$. Average baseline paw-pressure threshold across all groups was $150.0 \pm 9.7 \mathrm{~g}(\mathrm{n}=10$ for each group). Error bars are S.E.M. *: $\mathrm{P}<$ 0.05 , significantly different from the PBS-treated control.

injected into the back skin.

To examine the effect of an intraplantar injection of 1 pmol of MK-801 on the paw-pressure threshold in normal rats, MK-801 or PBS (0.1 $\mathrm{ml}$ each) was injected into the plantar surface of the hind paw and the paw-pressure threshold was measured at 15 minutes post-injection. The administration of MK-801 resulted in no significant change in the paw-pressure threshold as compared to the PBS-treated control group $(2.8 \pm 2.1 \%$ vs. $3.1 \pm 2.0 \%$ changes from baseline levels of $263.2 \pm 14.5 \mathrm{~g}, \mathrm{n}=10$ for each group).

\section{DISCUSSION}

The results of the present study indicate that local intraplantar injection of glutamate into the hind paw of normal rats evokes mechanical hyperalgesia, evidenced by a lowering of the paw-withdrawal threshold to pressure. This observation is consistent with the previous finding that an intraplantar administration of agonists for glutamate re- ceptor subtypes into the normal rat hind paw induced mechanical hyperalgesia. ${ }^{9)}$ The glutamate-induced mechanical hyperalgesia observed in the present study is likely receptor receptor-mediated since the stereoisomer Dglutamate produced no hyperalgesic effect. When previous studies showing the present presence of glutamate receptors on primary afferent axons, ${ }^{1,10,11)}$ are taken into consideration, glutamate-induced mechanical hyperalgesia seen in our study may be mediated in part by glutamate acting on its receptors on peripheral axons, and particularly on nociceptive fibers. Our data also indicate that local intraplantar injection, but not systemic administration, of NMDA receptor antagonist MK-801 attenuates FCA-induced mechanical hyperalgesia, suggesting a possible role of the peripheral glutamatergic system in the generation of inflammatory pain. This possibility is supported by the findings that the proportion of primary afferent axons immunostained for glutamate receptors increased during FCA-induced inflammation, ${ }^{3}$ and that glutamate levels increased in peripheral tissue inflamed 
with formalin. ${ }^{12)}$ Collectively, these results support the hypothesis that glutamate and its receptors present in peripheral tissue play a role in signalling pain in the normal and in the inflamed states.

It has been well known that nociceptive fibers in the inflammatory state are sensitized and their neuronal activity increases. ${ }^{13)}$ This increased nerve activity may cause the release of excess glutamate from peripheral nerve endings. This possibility, which is supported by a recent finding that, following peripheral nerve stimulation, glutamate levels increase in the hind paw extracellular space. ${ }^{14)}$ Peripheral activation of nociceptive fibers by glutamate has been suggested by an in vitro spinal cord-tail preparation in which peripherally peripherally-applied glutamate activates nociceptive afferent-mediated reflex. ${ }^{15,16)}$ Thus, it seems reasonable to hypothesize, when considered together with the fact that there are increases in both the numbers of glutamate receptor-labelled afferent fibers and the amount of glutamate in the hind paw during inflammation, that FCA-induced mechanical hyperalgesia observed in the present study might be the result of the increased nociceptive fiber activity evoked by excess glutamate during FCA-induced inflammation.

Our data show that local intraplantar injection of MK801 into the normal hind paw produces no change in the paw-pressure threshold. This seems due not to lack of glutamate receptors, but rather to lack of glutamates, in normal tissue, since local administration of glutamate into the normal paw evokes mechanical hyperalgesia. Alternatively, the amounts of glutamates present in the normal tissue is not sufficient enough to modulate the mechanical sensitivity of the hind paw.

In the present study, we observed that the lowered paw-pressure threshold during FCA-induced inflammation was reversed by MK-801, but not by CNQX, applied locally into the inflamed hind paw. This finding suggests that FCA-induced mechanical hyperalgesia is mediated via peripheral activation of NMDA, not by non-NMDA receptors. The previous study reported that both NMDA and non-NMDA receptor antagonists were effective in attenuating mechanical hyperalgesia following kaolincarrageenan-induced inflammation of the knee joint. ${ }^{7} \mathrm{~A}$ possible explanation for non-effectiveness of CNQX on FCA-induced mechanical hyperalgesia observed in the present study would be that peripheral expression of NMDA receptors" is higher than that of non-NMDA receptors during FCA-induced inflammation. Therefore, such activation of non-NMDA receptors is not sufficient enough to evoke mechanical hyperalgesia. This possibility is supported by the anatomical observation that the proportion of primary afferent axons immunostained for NMDA receptors is higher than that of AMPA or KA receptors in the hind paw inflamed with FCA. ${ }^{3)}$

An intraplantar injection of FCA into the rat hind paw has been shown to induce hyperalgesia to heat stimuli." There has been a report suggesting that thermal hyperalgesia following carrageenan-induced inflammation of the hind paw was mediated via activation of peripheral NMDA as well as non-NMDA receptors. ${ }^{6}$ ) Thus, one might expect that FCA-induced thermal hyperalgesia would be mediated via peripheral mechanisms acting on both NMDA and non-NMDA receptors although we have not tested on for this possibility matter. If this is the case, during FCAinflammation, the peripheral mechanism responsible for thermal hyperalgesia would be different from that for mechanical hyperalgesia, which is likely probably mediated by NMDA receptor activation as shown in the present study. A differential involvement of glutamate receptor subtypes in mediating different kinds of abnormal pain transmission within the spinal cord has been suggested. $^{\text {17-19) }}$

In conclusion, the present study demonstrates that local administration of glutamate into the rat hind paw induces mechanical hyperalgesia and that an intraplantar injection of MK-801, but not of CNQX, attenuates mechanical hyperalgesia following FCA-induced inflammation of the hind paw. The results suggest that NMDA, but not nonNMDA receptors play a substantial role in mediating the development of mechanical hyperalgesia induced in the inflamed paw following an intraplantar FCA injection.

\section{ACKNOWLEDGEMENTS}

This study was supported by grant from the Korea 
Health 21 R\&D Project, Ministry of Health and Wealth, Republic of Korea (HMP-98-N-2-0027) to Y.C.

This manuscript was accepted at Neuroscience Letters as of Nov. 2000.

Partly modified from Original version to fit in the submission format of Korean Journal of Anesthesiology.

\section{REFERENCES}

1. Cogggeshall RE, Carlton SM: Ultrastructural analysis of NMDA, AMPA and kainate receptors on unmyelinated and myelinated axons in the periphery. J Comp Neurol 1998; 391: 78-86.

2. Westlund KN, McNeill DL, Coggeshall RE: Glutamate immunoreactivity in the dorsal root axons. Neurosci Lett 1989; 96: 13-7.

3. Carlton SM, Coggeshall RE: Inflammation-induced changes in peripheral glutamate receptor populations. Brain Res 1999; 820: 63-70.

4. Cogggeshall RE, Cariton SM: Evidence for an inflammation-induced change in the local glutaminergic regulation of postganglionic sympathetic efferents. Pain 1999; 83: 163-8.

5. Davidson EM, Coggeshall RE, Carlton SM: Peripheral NMDA and non-NMDA glutamate receptors contribute to nociceptive behaviors in the rat formalin test. Neuroreport 1997; 8: 941-6.

6. Jackson DL, Graff CB, Richardson JD, Hargreaves KM: Glutamate participates in the peripheral modulation of thermal hyperalgesia in rats. Eur $J$ Pharmacol 1995; 284: 321-5.

7. Lawand NB, Willis WD, Westlund KN: Excitatory amino acid receptor involvement in peripheral nociceptive transmission in rats. Eur J Pharmacol 1997; 324: $169-77$.

8. Stein C, Millan MJ, Herz A: Unilateral inflammation of the hind paw in rats as a model of prolonged noxious stimulation: alterations in behavior and nociceptive thresholds. Pharmacol Biochem Behav 1988; 31: 445-51.
9. Zhou S, Bonasera L, Carlton SM: Peripheral administration of NMDA, AMPA or KA results in pain behaviors in rats. Neuroreport 1996; 7: 895-900.

10. Carlton SM, Hargett GL, Coggeshall RE: Localization and activation of glutamate receptors in unmyelinated axons of rat glabrous skin. Neurosci Lett 1995; 197: 25-8.

11. Kinkelin I, Brcker E-B, Koltzenburg M, Carlton SM: Localization of ionotropic glutamate receptors in peripheral axons of human skin. Neurosci Lett 2000; 283 : 149-52.

12. Omote K, Kawamata $T$, Kawamata $M$, Namiki $A$ : Formalin-induced release of excitatory amino acids in the skin of the rat hind paw. Brain Res 1998; 787: $161-4$.

13. Davis KD, Meyer RA, Campbell JN: Chemosensitivity and sensitisation of nociceptive afferents that innervate the hairy skin of monkey. J Neurophysiol 1993; 69: $1071-81$.

14. DeGroot JF, Zhou S, Carlton SM: Peripheral glutamate release in the hind paw following low and high intensity sciatic stimulation. Neuroreport $2000 ; 11$ : 497 502.

15. Ault B, Hildebrand LM: L-Glutamate activates peripheral nociceptors. Agents Actions 1993; 39: 142-4.

16. Ault B, Hildebrand LM: Activation of nociceptive reflexes by peripheral kainate receptors. J Pharmacol Exp Ther 1993; 265: 927-32.

17. Meller ST, Dykstra CL, Gebhart GF: Investigation of the possible role for carbon monoxide $(\mathrm{CO})$ in thermal and mechanical hyperalgesia in the rat. Neuroreport 1994; 5: $2337-41$.

18. Sluka KA, Jordan HH, Willis WD, Westlund KN: Differential effects of N-methyl-D-aspartate (NMDA) and non-NMDA receptor antagonists on spinal release of amino acids after development of acute arthritis in rats. Brain Res 1994; 664: 77-84.

19. Tal M, Bennett GJ: Neuropathic pain sensations are differentially sensitive to dextrorphan. Neuroreport 1994; 5: $1438-40$. 